\title{
La Marginalité Du Genre Dans Les Grandes Agglomérations En Côte d'Ivoire : Le Cas De La Prostitution De Luxe À Abidjan
}

\author{
M. Gueu Denis \\ Maître de Conférences en Criminologie \\ Université Félix Houphouët Boigny Cocody-Abidjan \\ UFR Criminologie, Abidjan
}

doi: 10.19044/esj.2016.v12n32p193 URL:http://dx.doi.org/10.19044/esj.2016.v12n32p193

\begin{abstract}
This study aims to understand and explain the phenomenon of luxury prostitution in Abidjan, to propose solutions. The research took place in the municipalities of Cocody and Marcory and were based on 30 participants. This is direct actors, including several prostitutes, their clients and indirect stakeholders such as the populations of these towns, some relatives and acquaintances of prostitutes and some structures' officials concerned, from which diverse information was collected. Data were collected using a literature review and questionnaires and then analyzed quantitatively and qualitatively. The results certainly indicate that several factors, namely, the crisis of the family unit, lack of employment, bad companions realize the luxury prostitution in Abidjan, but the most prominent is poverty. These factors resulting disastrous consequences, such as sexually transmitted diseases and HIV / AIDS to name a few.
\end{abstract}

Keywords: Luxury prostitution, poverty, HIV / AIDS, Abidjan

Résumé

Cette étude vise à comprendre et expliquer le phénomène de la prostitution de luxe à Abidjan, en vue de proposer des solutions. Les recherches ont eu lieu dans les communes de Cocody et Marcory et se sont appuyées sur 30 participants. Il s'agit d'acteurs directs, notamment quelques prostituées, leurs clients et d'acteurs indirects tels que les populations de ces communes, certains parents et connaissances des prostituées et quelques responsables de structures concernées auprès desquels des informations diversifiées ont été recueillies. Les données ont été recueillies à l'aide d'une étude documentaire et de questionnaires puis analysées d'un point de vue quantitatif et qualitatif. Les résultats obtenus indiquent certes que plusieurs 
facteurs, en l’occurrence, la crise des cellules familiales, le manque d'emploi, les mauvais compagnons rendent compte de la prostitution de luxe à Abidjan, mais le plus marquant est la pauvreté. De ces facteurs, découlent des conséquences désastreuses, notamment les maladies sexuellement transmissibles et le VIH/SIDA pour ne citer que ces exemples.

Mots clés : Prostitution de luxe, Pauvreté, VIH/SIDA, Abidjan

\section{Introduction}

En Côte d'Ivoire, la prostitution qui à l'origine était le fait de certaines ressortissantes ghanéennes et nigérianes, est de nos jours, avec la forte crise économique, aussi bien pratiquée par les ivoiriennes. Ce phénomène est d'une telle acuité que le sexe semble aujourd'hui être le moyen privilégié de survie et même de réussite sociale et les grandes structures d'hébergement, de restauration et de rafraîchissement ont toujours été le réceptacle d’individus aux situations financières assez confortables. Ce qui évidemment ne manque pas d'attirer le genre féminin dont des jeunes filles, notamment issues du milieu rural généralement en proie à de nombreuses difficultés en milieu urbain. Ceci semble faire de ces lieux les champs de multiples transactions basées sur le sexe et Abidjan, capitale économique de la Côte d'Ivoire, en est le lieu privilégié par excellence. Cette ville cosmopolite sous le joug «du plus vieux métier du monde » depuis des lustres a vu la recrudescence d'un nouveau type de prostitution au fil du temps : il s’agit de la prostitution de luxe. Celle-ci se pratique par le biais de réseaux de filles en agences d'escorte ou qui possèdent des appartements ou des villas et qui chassent plutôt dans la catégorie des hommes les plus riches et les plus les plus généreux. Les clients peuvent être des nationaux ou des visiteurs allogènes qui aiment bien être accompagnés pendant a durée de leur séjour.

Aujourd'hui encore, ce phénomène s’est accru avec l'appauvrissement généralisé de la société, appauvrissement qui d’ailleurs, a grandi le fossé entre les riches et les moins nantis.

En Côte d'Ivoire, à l'heure actuelle, le phénomène de la prostitution surtout celui de la prostitution de luxe prend de l'ampleur en s'inscrivant dans la vision de NORMANDEAU ( 1987) lorsqu'il affirme que l'industrialisation et l'intégration au marché économique mondial, tout en étant des facteurs de progrès sont parfois, à cause des mutations sociales brusques qu'elles produisent, des sources de déséquilibres et de conflits chez les adultes, comme chez les jeunes et les enfants. Ainsi, le processus d'acculturation, en s'accélérant et s'amplifiant, secrète des situations d'anomies, notamment en milieu urbain. Ce qui rend inévitable l'apparition de conflits de normes et de valeurs. Ces conflits, par la même occasion, 
favorisent des mécanismes ou des essais d'adaptation sociale, principalement chez les groupes vulnérables c'est-à-dire les enfants et les femmes. Le phénomène d’inadaptation sociale est selon SISSOKO (2006), le produit de ses conflits.

En réalité, la notion de prostitution renvoie généralement à des relations sexuelles entre hommes et femmes qui impliquent une transaction économique: pour les femmes, un service ou une prestation comprenant l'usage sexuel, pour les hommes, l'exercice d'une compensation ou d'une rétribution. Elle s'accommode à des comportements sexuels non acceptables socialement et est principalement considéré comme étant un espace déviant, voir immoral.

La présente étude vise à explorer le processus par lequel la gente féminine s'abandonne à la prostitution et en particulier celle dite de luxe à Abidjan. En effet, le portrait général de la prostitution semble impossible à saisir dans toute sa complexité socio-culturelle, mais elle permet en amont de situer le chercheur dans une analyse de la marginalité du genre, notamment en milieu urbaine en Côte d'Ivoire. De plus en plus, la société ivoirienne enregistre de nouvelles gammes de déviances et de marginalités qui enrichissent le décor de la criminalité. Apparemment la recherche du gain à travers la prostitution est le plan plus visible qui semble cacher une pléthore de comportements et d'actes parfois en contradiction avec les normes et les valeurs que la société ivoirienne prône.

Une telle étude pour nous, a ses raisons d'être au niveau social et scientifique. Au niveau social, à cause de la recrudescence des maladies liées au sexe qui préoccupent les décideurs. Au plan scientifique, pour éclairer le monde scientifique sur les rapports de causalité entre le trio : prostitution de luxe, le contact avec certaines autorités gouvernementales et la criminalité organisée. Plusieurs études continuent d'explorer ce domaine mais nous voulons à notre niveau toucher aux aspects criminogènes de la prostitution de luxe à Abidjan.

Cet article scientifique comporte trois parties essentielles: une première partie consacrée à la méthodologie employée, une deuxième partie comportant les résultats de l'enquête et une troisième partie faisant les recommandations et la conclusion.

\section{Méthodes}

Une étude scientifique découle toujours d'un constat concernant un problème ou une problématique. C'est pourquoi, le problème qui a orienté notre pensée à cette étude se situe dans la question essentielle suivante : pourquoi la prostitution de luxe prend -t- elle de l'ampleur à Abidjan ?

Comme techniques de recueil des informations, nous avons eu recours d'abord à la documentation consistant à la lecture des différents 
écrits relatifs au sujet de la prostitution. Ensuite, en collaboration avec certains de nos étudiants notamment l'étudiant N'GORAN KOUAME CONSTANTIN (en master 2 de criminologie), nous avons administré des questionnaires à une catégorie diversifiée d'individus choisis à l'aide d'un échantillonnage arbitraire faute de base de données fiables sur le phénomène. La commune de Cocody et celle de Marcory ont servi de terrain de notre enquête. Le choix de ces communes se justifie par le taux élevé de lieux de «joie » à travers les bars climatisés, les espaces gastronomiques, les cybercafés, les maquis etc. Il convient de signaler également que ces deux communes choisies attirent la plupart des artistes chanteurs, en l'occurrence les « DJ ».

Pour une meilleure analyse et interprétation des résultats, les théories de la dyssocialité, du contrôle social de HIRSCHI et la théorie de l'anomie de MERTON ont été d'un apport important. Au fond, il s'agit d'interpréter les liens sociaux en termes de relâchement des attachements aux valeurs et aux normes qui déterminent le système social en côte d'ivoire. La société ivoirienne en général et particulièrement celle de la capitale économique connaît de plus en plus un déséquilibre socio-culturel profond et complexe qui nécessite une réflexion critique en vue de dégager un meilleur remède.

La taille de l'échantillon qui est de trente (30) personnes nous parait faible mais cela répond à un souci d'objectivité. Nous voulons éviter de tomber dans un pluralisme et un flux inutile de population qui nous éloignerait de la rigueur scientifique recherchée.

\section{Résultats}

\section{Manifestations du phénomène}

Il s'agira pour nous ici d'étudier les signes, de voir comment se manifeste la prostitution féminine de luxe à Abidjan.

\section{Typologie des acteurs impliqués dans la prostitution féminine de luxe}

Tableau 1 : Répartition des acteurs impliqués dans la prostitution de luxe selon leur

\begin{tabular}{|c|c|c|c|}
\hline \multicolumn{2}{c|}{ typologie } \\
\hline \multirow{2}{*}{$\begin{array}{c}\text { TYPOLOGIE } \\
\text { Acteurs } \\
\text { directement } \\
\text { impliqués }\end{array}$} & Réceptionnistes d'hôtels & EFFECTIF & POURCENTAGE \\
\cline { 2 - 4 } & Gérants de bars & 05 & 8,92 \\
\cline { 2 - 4 } & Macs & 06 & 10,71 \\
\cline { 2 - 4 } & Prostituées de luxe & 04 & 7,14 \\
\cline { 2 - 4 } & Gérants de restaurants & 17 & 30,35 \\
\hline \multirow{3}{*}{ Acteurs concernés } & Autorités municipales & 04 & 7,14 \\
\cline { 2 - 4 } & Agents police mondaine & 04 & 7,14 \\
\cline { 2 - 4 } & Riverains & 04 & 7,14 \\
\hline & Total & 12 & 21,46 \\
\hline
\end{tabular}

Source : Notre enquête 
Il en ressort du tableau ci-dessus que l'effectif de l'échantillon des acteurs impliqués dans la prostitution féminine de luxe est de cinquante-six (56) individus. Dans cet effectif, les acteurs concernés $(35,74)$. Cette disparité au niveau de cette répartition s'explique par la disponibilité des acteurs directement impliqués.

Compte tenu de leur activité, cette catégorie d'individu profite du voisinage avec les prostituées de luxe avec des clients moyennant une rémunération de ceux-ci (prostituées de luxe et client).

Tableau 2 : Distribution de la variable âge des prostituées à la solde des acteurs Impliqués

\begin{tabular}{|c|c|c|c|c|c|c|c|c|}
\hline \multirow{2}{*}{$\begin{array}{c}\text { Typologie } \\
\text { Age (Année) }\end{array}$} & \multicolumn{3}{|c|}{ Exploiteurs professionnels } & \multicolumn{4}{c|}{ Démarcheurs } \\
\cline { 2 - 9 } & $\begin{array}{c}\text { Réceptionnistes } \\
\text { d'hôtel }\end{array}$ & \multicolumn{3}{|c|}{ Macs } & \multicolumn{2}{c|}{ Gérants de bars } & \multicolumn{2}{c|}{ Gérants de restaus } \\
\cline { 2 - 9 } & Effectif & $(\%)$ & Effectif & $(\%)$ & Effectif & $(\%)$ & Effectif & $(\%)$ \\
\hline$[17-20]$ & 01 & 8,33 & 01 & 8,33 & 02 & 16,66 & 00 & 00 \\
\hline$[20-25]$ & 02 & 16,66 & 01 & 8,33 & 01 & 8,33 & 01 & 8,33 \\
\hline$[25-30]$ & 01 & 8,33 & 00 & 00 & 01 & 8,33 & 01 & 8,33 \\
\hline$[30-35]$ & 00 & 00 & 00 & 00 & 00 & 00 & 00 & 00 \\
\hline $\begin{array}{c}{[35 \text { et }} \\
\text { plus [ }\end{array}$ & 00 & 00 & 00 & 00 & 00 & 00 & 00 & 00 \\
\hline Total & 04 & 33,33 & 02 & 16,66 & 04 & 33,33 & 02 & 16,68 \\
\hline
\end{tabular}

Source: Notre enquête

L'enquête montre à travers ce tableau que la prostitution de luxe des mineurs est relativement élevée au niveau des gérants de bars. Cela s'explique par le fait que les adolescentes sont beaucoup attirées par les lieux publics en effervescence. La recherche du gain facile pour ces filles venues tenir compagnie à des personnes aisées en quête de conquêtes pour soulager leur libido est parfaite occasion pour elles. Les bars ou casinos sont des lieux indubitables pour s'affranchir de la disette économique en croisant des hommes fortunés.

D'après les informations recueillies, les réceptionnistes d'hôtel et les gérants de bars accusent un fort taux d'exploitation sexuelle des jeunes filles prostituées de luxe comprises dans la tranche d'âge dans la fleur de l'âge est beaucoup plus prisée par les clients.

La tranche de 25 à 30 ans fait l'objet de peu de demandes $(25,03)$. Par contre les deux dernières tranches d'âge, c'est-à-dire celle de 30 à 35 ans et celle de 35 ans et plus enregistrent un pourcentage nulle $(0 \%)$. Cela s'explique par le fait que dès l'âge de 30ans, les prostituées de luxe vont plutôt opter pour leur économie. Elles vont donc commencer à avoir leur propre carnet d'adresses de clients et faire carrière seule. Indépendantes, d'autres prostituées de luxe vont même encadrer d'autres nouvelles jeunes filles qui voudront faire leur premier pas dans le monde de la perversion et de la débauche en quête elle aussi de finance.

En outre, les prostituées de luxe sont en majorités d'origine ivoirienne (35,29 \%). Après elles, viennent les burkinabés et les libériennes. 
Nous notons une forte présence des autochtones dans la prostitution féminine de luxe et les autres sont de nationalités diverses en particulier africaine de la sous-région. Elles quittent leur pays d’origine à la recherche d'un mieux-être, malheureusement elles sont confrontées à une autre réalité.

Selon M'Baye « la situation de plus en plus compliquée dans mon pays m'a fait quitter les parents pour un lendemain meilleur et leur venir en aide plus tard. Venus chercher du boulot en Côte d'Ivoire, une amie m'a mise dans ce milieu qui me fait gagner aujourd'hui mon pain. Au début j'étais hésitante mais après j'ai vu que ça me rapportait gros et $j$ 'ai pris à cour ce métier de luxe ".

Dans le cadre de notre enquête, nous avions eu à faire la rencontre d'une femme originaire du Maghreb qui représente 5,91\% de notre effectif. Il s’agit d'une marocaine qui a bien voulu répondre un tant soit peu à nos questions.

Sur les prostituées de luxe interrogées, nous avons constaté qu’elles sont toutes d'un niveau d'instruction assez élevé. 6 soit 35,29\% des enquêtées ont atteint le cycle secondaire et 11 soit $64,71 \%$ sont arrivées au cycle supérieur. Malgré ce fort rendement d'instruction, un faible niveau d'employabilité est quand même enregistré. Ce qui concoure à leur forte intrusion dans le monde prostitutionnel. Mandjo Estelle se prononcera sur cette question en disant : «j’ai eu la licence en espagnole et en quête de travail pour m’assumer, j'ai rencontré une amie avec qui nous sommes allées travailler dans un bar. Au fil du temps, nous avions fait connaissance avec une camarade de la 1ere année à l'université qui venait de temps à autre dans notre bar en compagnie de certaines personnalités aisées. Chemin faisant, nous avions tout fait pour l'approcher et avoir son tuyau qui la mettait dans le luxe indescriptible. C'était une Escort-girl par le biais d'un propriétaire de bar bien connu dans le milieu du showbiz. Quelques temps plus tard, elle nous fit rentrer dans son « réseau »

Facteurs explicatifs de la prostitution féminine de luxe

L’Affaiblissement du contrôle social

Tableau3 : Situation des parents

\begin{tabular}{|c|c|c|}
\hline Situation matrimoniale & Effectifs & Pourcentage \\
\hline Ensemble & 10 & 58,82 \\
Divorcé & 05 & 29,41 \\
Décédé & 02 & 11,77 \\
Total & 17 & 100 \\
\hline
\end{tabular}

Source : Traitement de notre enquête

La prostitution féminine de luxe provient d'abord de familles où les parents vivent ensemble (58,82 \%), puis de famille où les parents sont divorcés $(29,41 \%)$. Nous enregistrons un pourcentage faible de familles de 
prostituées où les parents sont décédés (11,77\%). Le tableau nous montre que la plupart des prostituées de luxe sont issues de familles biparentales. Malgré la présence du père et de la mère vivant toujours ensemble, elles ont choisi ce métier soit pour s’abandonner au gain facile avec de multiples partenaires sexuels en vue de satisfaire leurs besoins effrénés de luxe, soit par fantasme (les nymphomanes).

Geneviève, l'une des prostituées de luxe rencontrée au quartier millionnaire de Yopougon s'exprimait en ces termes: "mes parents de situation modeste répondaient toujours à mes aspirations mais mon gout sans cesse pour le sexe m'a fait fuir la maison. J'avais cette passion de rencontrer encore plus de mecs pour atteindre mon paroxysme sexuel. Ça ne m'a donc pas gêné de rentrer dans le milieu de la prostitution de luxe ".

Les propos de cette jeune fille, montre l'image négative qu'elle a pour l'autorité parentale, traduisant ainsi sa volonté manifeste de se défaire de l'étau de séquestration selon elle dans lequel ses parents l'avaient fait prisonnière. Et cela au mépris des valeurs de l'éthique et de la morale, elle s'est adonnée à la prostitution. Cette forme de réaction particulière d'emprise des parents, est intériorisée par les enfants et crée un esprit de vengeance qui ne trouve solution que dans un vice, ici notamment celui de la prostitution. Cette forme de réaction particulière d'emprise des parents, est intériorisée par les enfants et crée un esprit de vengeance qui ne trouve solution que dans un vice, ici notamment celui de la prostitution.

Quant aux prostituées issues de familles monoparentales le père ou la mère étant toujours absent, cela va constituer un manque dans l'éducation de la jeune fille qui va donc s’affligé à un groupe de pairs à la longue. Ce groupe sera un modèle pour celle-ci et va déterminer fortement son adhésion future dans le monde prostitutionnel. Cela est identique pour les prostituées dont les deux parents sont décédés.

"Chacune n'a pas les mêmes chances de survie dans ce monde facile pour certaines et difficile pour d'autres, il faut vivre avec ce que la nature nous a offert de mieux " : lâchera ironiquement dame Momo au passage comme pour justifier son acte prostitutionnel depuis la mort de ses deux parents lorsqu'elle n'avait que 14 ans.

En outre, la carence éducative des parents, le déséquilibre des rôles parentaux, la carence affective, l'abandon précoce des enfants qui seront livré à eux-mêmes, la détermination, le rejet des enfants, les conflits intrafamiliaux, la violence familiale, le désir de prouver aux parents démissionnaires sa capacité à prendre son indépendance, sont des variables qui vont expliquer la prostitution de luxe de quelques jeunes filles à Abidjan.

La carence éducative des parents s'explique par un manque ou une faiblesse au niveau de l'éducation des enfants. Le manque par exemple d'affection, de chaleur au sein de la famille (père-mère-enfant) est du soit au 
nombre un peu trop élevé des enfants en charge (familles nombreuses), soit à la recomposition de la famille monoparentale.

Par famille nombreuses, il faut entendre des familles qui ont leur sein au moins quatre (4) enfants. Pour ces familles, il est très difficile pour les parents d'accorder la même affection aux enfants. Etant nombreux, il va s'en dire que chaque enfant recevra moins d'affection, moins d'attention et de soins qu’il aurait reçu si la fratrie avait été inférieure à ce nombre.

Par famille recomposée, il faut entendre une famille où les deux ou l'un des conjoints a déjà connu une vie de couple, en ayant plus ou moins des enfants à l'appui. Dans cette nouvelle famille, lorsqu'un parent n'accorde pas assez d'importance aux enfants hors couple, cela a une influence sur la vie en famille. C'est pour montrer combien le sentiment d'être mal ou pas aimée du tout pourrait conduire certaines personnes à des actes déviants tels que la drogue, la délinquance juvénile ou la prostitution. Il faut noter qu’en plus du désintérêt du père, il faut compter sur le mépris de la belle-mère qui, soucieuse du bonheur de ses propres progénitures, fera tout son possible pour écarter les enfants de son époux en leur rendant la vie impossible.

Par famille monoparentale, il faut voir les familles avec un père ou une mère comme seul parent. Ici, le plus souvent, le parent étant à bout de force permet un laisser-aller dans sa famille. Et ceci ne peut qu'entrainer des vices dans sa famille. Et ceci ne peut qu'entrainer des vices dans sa famille car il adopte un style permissif qui laisse les enfants décider de leur sort.

En effet, ceux-ci étant déjà fragilisés par la séparation des parents, sont en général désespérés ; surtout quand la mère ne vit plus ou qu'elle est séparée totalement d'avec ses enfants qui ne bénéficient d'aucun apport extérieur venant d'autres parents tels que l'oncle, la tante, le cousin ou les grands parents. Ainsi, senties délaissées, ces jeunes filles qui sont le plus souvent des mineures, vont foncer tout droit dans la prostitution, d'autant plus que la tentation ne se fera pas rare avec ces hommes âgés, qui sont prêt à dépenser d'autant plus d'argent rien que pour assouvir leur appétit sexuel.

Aussi, nous avons la démission des parents qui parfois contribue à l'incitation des filles à la prostitution. En effet pour beaucoup de parents, le rôle de père se limite à la nourriture, au logement et à la scolarisation de leur progéniture. Les autres dépenses ne les regardent aucunement puisqu’ils sont à la retraite pour la plupart. Ainsi, la jeune fille va faire face aux dures réalités de la vie jusqu'à succomber à la tentation souvent dictée par l’influence de groupe de pair, en occurrence la mauvaise compagnie.

\section{Situation socio-économique}

La prostitution occasionnelle ou la prostitution professionnelle est souvent le fait des prostituées issues des familles pauvres ou incapables de les contrôler. Beaucoup d'entre elles sont à la recherche de leur pain 
quotidien. Peu sont celles qui se prostituent par plaisir. Elles cherchent de l'argent pour subvenir à leurs besoins et à ceux de leurs familles.

Par contre, la prostitution de luxe existe autant plus dans la classe favorisée que démunie selon notre enquête. Cependant, il convient de noter que la prostitution de luxe, bien qu'ayant pour facteur principal l'indigence économique mérite aussi d’être expliquée à travers l'insuffisance d'aide de l'Etat citée plus haut et la cherté de la vie.

La cherté de la vie quant à elle, est un problème très crucial dans le district d'Abidjan. En effet, depuis la crise qu'a connue la Côte d'Ivoire en septembre 2002, les populations du nord et celles de l'ouest, pour une question de sécurité ont décidé de fuir vers la capitale économique. Ceci ayant créé l'exode rurale massif provoquant ainsi un surpeuplement au niveau du district d'Abidjan. Depuis lors, on assiste à une montée des prix des denrées alimentaires et même des logements, Ce qui va rendre systématiquement cette ville de plus en plus difficile.

Tableau : Personnes prises en charge par les prostituées de luxe

\begin{tabular}{|c|c|c|}
\hline Personnes à charge & Effectifs & Pourcentage \\
\hline 0personne & 09 & 52,94 \\
1 à5 personnes & 06 & 35,29 \\
5 à10 personnes & 02 & 11,77 \\
Total & 17 & 100 \\
\hline
\end{tabular}

Source : Traitement de notre enquête

A propos du nombre de personnes prises en charge par les prostituées de luxe, l'étude nous révèle par le biais des résultats du tableau, que plus de la moitié des prostituées de luxe (52,94 \%) ne prennent personnes en charge. Cela s’explique par le fait que bon nombre de prostituées de luxe vivent déjà dans des conditions favorables propices à leur situation familiale. L'autre moitié (35, $29 \%)$ et $(11,77 \%)$ sont respectivement la classe moyenne et défavorisée.

Nous constatons qu'en Afrique et particulièrement en Côte d'Ivoire, que c'est la classe démunie qui a plus de personnes en charge. Les prostituées de luxe figurant dans ce dernier cas vont donc exercer ce métier pour subvenir à leurs besoins et aux besoins des personnes qu'elles ont à leur charge.

Selon les propos recueillis de Yasmine: «j’ai été obligée de me prostituer pour scolariser mes deux frères qui n’avaient que moi comme seul soutien au décès des parents. Malheureusement, nous avons été abandonnés très tôt aux mains d'un oncle polygame qui nous demandait à chaque fois de nous débrouiller pour gagner notre pitance quotidienne car il nous abritait gratuitement sous son toit et c'est tout ce qu'il pouvait faire pour nous. J'ai dû dès lors arrêter l'école en classe de seconde $C$ pour prendre en charge mes frères et moi ». 
Mis à part cet état de fait, nous avons le revenu moyen par habitant qui est relativement bas. L'accès au soin, à l’école, au logement, à l'emploi constitue encore une préoccupation majeure pour nos dirigeants politiques.

En effet, nous constatons qu'il y a peu de centre de santé ou de systèmes de prises en charge pour prostituées. Les médicaments coutent chers à l'hôpital, à la pharmacie et sans argent aucun soin ne peut être administré. Nous nous rendons compte que l’Assurance Maladie Universelle prônée par les autorités dirigeantes n’est pas totalement opérationnelle.

Lorsque nous analysons les facteurs d'incitation (besoins des prostituées de luxe) à la pratique de la prostitution de luxe, il en ressort que l'argent est le moteur de cette pratique (14,06 \%) et comme l'affirme encore Maya : "L'argent est le facteur clé de la réussite sociale de nos jours, sans argent on ne peut rien et on vaut rien ".

Celles qui optent pour le travail sont peu négligeables (23,53 \%). Et comme le monde du travail n'est pas facilement accessible à tous de nos jours, certaines succombent aux vices de la prostitution qui est un moyen de gain rapide sans hésitation. Celles qui pensent à un mariage et donc à une vie de couple sont minoritaires (11,76 \%).

Les prostituées de luxe dont les besoins sont autres, c'est-à-dire non mentionnés tels que les tenues vestimentaires, le plaisir sexuel, les multiples partenaires, les matériels ou les objets de luxe sont estimés à (17,65 \%).

Un agent de la police mondaine dans une causerie amicale avec d'autres étudiants nous dit ceci : «certaines filles, pour faire face à toutes ces difficultés, ont opté pour le monnayage de leur charme contre quelque chose, d'autres s'en sortent avec des activités tels que les cabines téléphoniques, les serveuses dans les maquis et les restaurants et les enfants qu'elles ont en charge. Celles qui sont scolarisées, le font de manière périodique et les déscolarisées font de cela leur activité principale. Dans tous les cas, chaque prostituée a son motif valable et les prostituées de luxe s'en sortent beaucoup plus que les autres types de prostituées ".

\section{Conséquences et proposition de solution}

\section{Maladies sexuellement transmissibles}

Ces maladies sont pour la plupart les premiers risques dans la prostitution. Car par l'influence de certaines personnes, du fait de leur fortune, les prostituées de luxe souvent n’exigent pas les préservatifs, entretiennent des rapports non protégés avec ces derniers qui paient chers la nuit ou la passe. Ceci, crée des risques de contraction des maladies telles que les MST et les IST, voire même le VIH/SIDA. Certaines maladies telles que la gonococcie et autres, sont en général, des maladies curables et sans véritable danger sur le fonctionnement de l'organisme, si elles sont vite découvertes et traitées aussitôt. Mais dans le cas contraire, elles peuvent 
endommager terriblement l'organisme et même entrainer des dysfonctionnements graves au niveau de l'appareil reproducteur.

En Afrique et plus particulièrement en Côte d'Ivoire, le SIDA est l'une des maladies les plus dangereuses après le paludisme. Notons que cette maladie demeure une maladie incurable et représente, l'une des principales causes de mortalité après le paludisme.

Généralement, la prostitution n’a d'effet que sur les deux principaux acteurs c'est-à-dire, la prostituée et le client. Les risques de contamination de maladies telles que le VIH/SIDA ne sont pas négligés car à cause des nombreux rapports sexuels avec plusieurs partenaires, les différents acteurs, par manque de précaution peuvent être infectés et en mourir.

\section{Consommation de produits psychotropes}

La consommation de la drogue ou de l'alcool est un passage obligatoire pour la réussite des activités sexuelles pour les prostituées de luxe. C'est un moyen plus sûr pour contourner la peur et la honte. En effet, la prostitution est un phénomène qui cache d'autres pratiques déviantes. Dans ce milieu, la consommation de l'alcool et de la drogue joue un rôle indispensable, tant chez les mineures que chez les majeures prostituées. Les endroits fréquentés par les mineures prostituées sont en général les bars et les maquis. Selon la culture adoptée dans ces endroits, l’ampleur de la puissance de la poche d'un individu se caractérise par le nombre de boissons commandées. Et certaines personnes, pour se faire remarquer, vont jusqu’à jeter des billets de banque sur des disc-jockeys (DJ) et demandent qu'on remplisse leurs tables de bouteilles de boissons. En ce qui concerne les mineures prostituées de luxe, dans les débuts, elles prennent l'alcool et dans l'inconscience, se livrent à des clients, qui après avoir consommé une dose relativement élevée, cherchent à satisfaire leur libido. Après la première expérience, elles s’y habituent et deviennent des alcolo-dépendantes. Que ce soit des prostituées mineures de luxe ou des majeures, la consommation de l'alcool ou de la drogue produit toujours des effets négatifs sur la santé de l'individu. Consommé dans les débuts, l'alcool provoque le vertige, les vomissements, de vives migraines, mais sans réel danger sur le fonctionnement du consommateur. Mais plus le taux d'alcool devient important dans le corps, plus il crée un dysfonctionnement dans l'organisme. On observe à ce niveau chez les hommes, l'asthénie sexuelle (état de fatigue et d'épuisement), l’inhibition de l’organisme, la diminution de la sécrétion de sperme, du nombre de spermatozoïdes et leur virilité. On observe aussi parfois une absence de sperme et la chute important des poils du pubis, la gynécomastie (développement anormal des glandes mammaires), l'atrophie testiculaire et l'impuissance sexuelle. Chez la femme, nous avons des troubles au niveau de l'ovulation et des menstrues, la ménopause précoce. 
Dans le cas de la drogue, dans notre société, tout individu à un moment de son existence peut basculer, pour une raison ou une autre dans la toxicomanie. Toutefois, certains individus, pour des raisons liées à la fois à leur personnalité et à leur environnement semblent être plus exposés. Ces personnes viennent de toutes les classes sociales. Concernant le domaine de la prostitution, la drogue est l'un des trafics qui rapporte le plus aux vendeurs. La drogue est tout comme l'alcool, un moyen pour certaines personnes de se défaire de la peur et d'affronter la vie. C'est malheureusement un véritable danger pour ces filles en difficultés mentales. En effet, à long terme, la drogue peut conduire à une affection du système cardiovasculaire, du foie, des reins, entraîner le diabète, le glaucome (maladie des yeux) et surtout provoquer une dépendance de celles-ci.

\section{Conclusion}

$\mathrm{Au}$ terme de cette étude, il convient de situer l'ampleur de la prostitution féminine à Abidjan. Il suffit de parcourir certaines rues comme la rue des jardins au Vallon à cocody, entre 22 heures et 4 heures du matin pour se rende compte de l'évidence. Des adolescentes jusqu'aux femmes adultes, on assiste à une lutte sur les clients dont les plus prisés sont les européens.

Parler de solution pour le criminologue que nous sommes, serait trop prétentieux, mais pour atténuer une telle pratique, il faut agir sur la société elle-même. Pour nous, Il faut par ailleurs pouvoir trouver des moyens adéquats entre la formation et l'emploi et instaurer à l'école une période de campagne de sensibilisation. Cela pourra permettre surtout aux jeunes filles d’avoir une idée sur la sexualité et les risques encourues par celles-ci lors des rapports trop précoces. Ainsi, associer les parents à l'éducation de leurs enfants tout en adoptant un comportement exemplaire face à leurs progénitures serait une possibilité importante dans le processus de resocialisation de ces prostituées. Ceci dépend également des séances d'échanges entre éducateurs, élèves et ONG qui œuvrent dans la lutte contre les MST (maladies sexuellement transmissibles), le VIH/SIDA et la toxicomanie.

Notons que le père et la mère qui sont les piliers principaux de la famille, doivent en outre créer les moyens d’épanouissement de leurs progénitures et leur faire comprendre le danger de la prostitution. Nous insistons sur l'éducation familiale, car elle est déterminante en amont dans une société ivoirienne où le sexe n’est plus un tabou. Il faut expliquer les choses les plus intimes et surtout touchant au sexe aux enfants.

Dans notre enquête, il s’est trouvé que certains parents encouragent leurs filles soit aux mariages précoces soit à la prostitution, ces deux pratiques ayant le même dénominateur commun qui est celui du profit et du 
gain facile faisant de la jeune fille un appât servant de réserve à la prise en charge illicite des parents. Il faut arrêter ces pratiques, surtout le concours du Ministère de la famille et des enfants serait utile. Même, si la crise de la cellule familiale devient de plus récurrente, il convient d'aller au-delà de la simple sensibilisation et appliquer des textes du code pénal en ce qui concerne la pratique de la prostitution.

\section{References :}

1. ASSI A. R. (2002). La prostitution informelle des mineurs issus du milieu rural dans les bars et réceptifs hôteliers en côte d'ivoire : le cas de la ville d'Adzopé. Mémoire de maitrise en criminologie (document non publié), UFHB.

2. DURKHEIM E. (1992). Les règles de la méthode sociologique. PUF, Paris.

3. GORDFROY P. (2002). De la problématique de la prostitution à la question de la DDASS dans le département du var (rennes), mémoire de l'école national de santé publique, PUF, Paris.

4. GRAWITZ M. (2001). Méthode des sciences sociales. Paris : Dalloz, $11^{\text {ème }}$ édition.

5. GRAWITZ M. (2004). Lexique des sciences sociales. Dalloz, $8^{\text {ème }}$ édition, Paris

6. KAZON D. (2009). L'exploitation sexuelle des enfants dans les maquis, bars et hôtels d'Abidjan. Thèse de doctorat en criminologie (non publié). UFHB.

7. N'DA P. (2002). Méthodologie de la recherche : de la problématique de la discussion des résultats, EDUCI, Abidjan.

8. SELOSSE J. (1997). Adolescences, violences et déviances. Édition matrice, Paris

9. SISSOKO A. (1995). La délinquance juvénile à Abidjan, conférence, Abidjan.

10. SISSOKO A. (2005). Les jeunes filles déscolarisées à Abidjan, logique d'adaptation et dynamique sociale en milieu urbain. Revue africaine de criminologie. EDUCI, Abidjan. $\mathrm{N}^{\circ} 2$ P.62-84.

11. SISSOKO A. (2006). Communication sur la conception culturelle de l'enfant en Afrique grand Bassam : revue africaine de criminologie, EDUCI, Abidjan.

12. TEKA E. (1999) Étude du phénomène de la prostitution des mineurs en milieu urbain. Mémoire de maitrise en criminologie, de document non publié. Université de cocody-Abidjan. 